\title{
RUBELLA OUTBREAK IN WESTERN SYDNEY, SPRING 1995: IMPLICATIONS FOR RUBELLA SURVEILLANCE AND CONTROL
}

\author{
Tim Heath ${ }^{a, b}$, Epidemiology Registrar, Master of Applied \\ Epidemiology Program \\ Anthony Capon ${ }^{a}$, Director, Western Sector Public Health Unit \\ Christine Roberts ${ }^{b}$, Director, Master of Applied Epidemiology \\ Program \\ a. Western Sector Public Health Unit. \\ b. National Centre for Epidemiology and Population Health.
}

In the spring of 1995 there was a Statewide rubella outbreak in $\mathrm{NSW}^{1,2}$ which presented the opportunity to compare the characteristics of laboratory confirmed and clinically diagnosed rubella. This article reports on the comparison.

Rubella is usually a mild febrile illness of childhood, but maternal infection during early pregnancy and subsequent foetal infection can result in a range of devastating developmental malformations - including mental retardation, deafness, blindness and congenital heart disease - which are broadly referred to as Congenital Rubella Syndrome (CRS). Unfortunately, there is no effective treatment for intrauterine rubella infection, so CRS prevention depends primarily on immunisation strategies. In planning these strategies, we rely principally upon surveillance of rubella disease and immunity.

In NSW, rubella is notifiable only for diagnoses confirmed by culture or serology. School principals and child care directors are also required to report suspected cases, but these reports must be serologically confirmed to satisfy notification criteria. By contrast, the National Health and Medical Research Council (NHMRC), in addition to laboratory-based notification, recommends a clinical surveillance definition for notification by doctors, when cases are linked to other laboratory-confirmed rubella diagnoses. Serologically-based surveillance of rubella is highly specific, but it is less certain whether it accurately reflects risk groups and allows useful intervention.

\section{METHODS}

During 1995 the Western Sector Public Health Unit (WSPHU) routinely recorded unsolicited reports of clinically diagnosed rubella. A 'clinical' case was defined as: (1) an acute febrile illness associated with a generalised maculopapular rash reported by a medical practitioner, child care director or school principal; (2) the illness had been clinically diagnosed as rubella by a medical practitioner; (3) no confirmatory serology or cultures had been performed; and (4) the illness did not meet the clinical criteria for measles notification.

The same data were collected for these clinical reports as is routinely collected for laboratory-confirmed rubella notifications, and these data - age, sex, occupation, language spoken at home, geographic distribution, and delay from onset date to notification date - were retrospectively compared. A 'serologic case' (there were no culture-positive notifications) was defined as a case with a single high titre rubella IgM, or a fourfold rise in serum IgG, in the absence of recent immunisation. All cases were resident in Western Sydney or Wentworth Health Areas. Analyses were performed using Epi Info 6. The chi-square test for contingency tables was used for categorical analyses, and the Mann-Whitney U test was used for non-parametric,

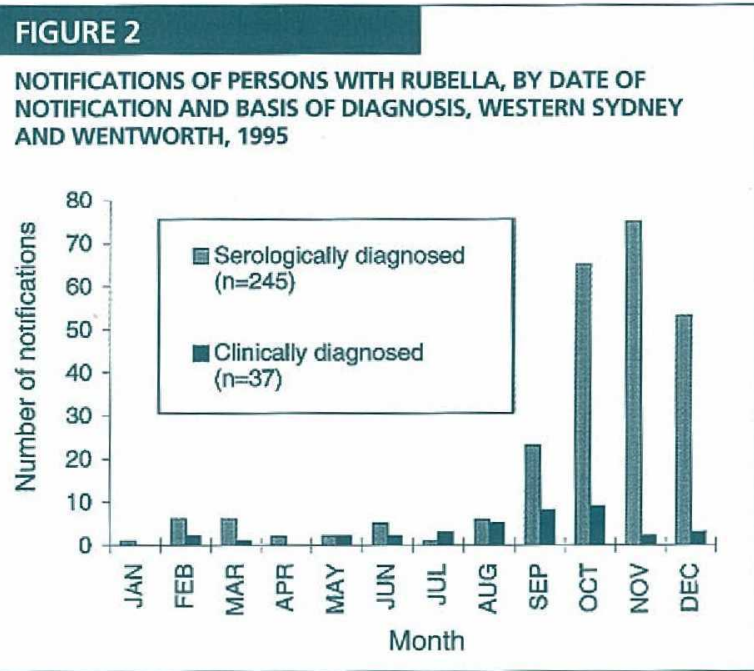

continuous data. Notification rates were calculated using the Australian Bureau of Statistics estimated mid-year populations for 1994.

\section{RESULTS}

During 1995 there were 245 serologically confirmed notifications of rubella from Western Sydney and Wentworth Health Areas - a crude notification rate of 26 cases per 100,000 for both Areas combined. One hundred and eighty-five of these notifications occurred during the September-November quarter, compared to seven during the same period in 1994 . There were 30 notifications among women aged 15 to 44 years - a rate of $6.4 / 100,000$. The serologic notification rates in Wentworth and Western Sydney Areas were 17/100,000 and 30/100,000 respectively. There were 37 clinically diagnosed cases during 1995. Clinical case reports peaked earlier than serologic cases (Figure 2).

The median age of serologic cases was significantly older (19 years) than clinical cases ( 8 years; Mann-Whitney $p<0.001$ ). Serologic cases were predominantly male ( 77 per cent), in contrast to clinical cases of whom 54 per cent were male $\left(\chi^{2}\right.$ $\mathrm{p}=0.01$ ). This male predominance among serologic cases was most marked between the ages of 15 and 25 years (Figure 3). However, there was no difference between the sex distribution of serologic and clinical cases after controlling for age.

Occupation was recorded for 20 per cent (49/245) of serologic cases and all clinical cases. Fourteen per cent of serologic cases (7/49) were in child care or primary school, compared to 81 per cent (30/37) of clinical cases. Almost all serologic (44/45) and clinical cases (23/24) were from English speaking homes. The median time from illness onset to receipt of notification was significantly longer for serologic cases (19 days) than clinical cases ( 3 days; Mann-Whitney $\mathrm{p}<0.001$ ).

\section{DISCUSSION}

Our serologically derived surveillance data suggested that this outbreak of rubella occurred essentially in young adult males. Serologic data also implied that children of both sexes aged $<5$ years old were protected, as might be 


\section{FIGURE 3}

AGE AND SEX DISTRIBUTION OF PERSONS NOTIFIED WITH SEROLOGICALLY CONFIRMED RUBELLA IN WESTERN SYDNEY AND WENTWORTH, 1995

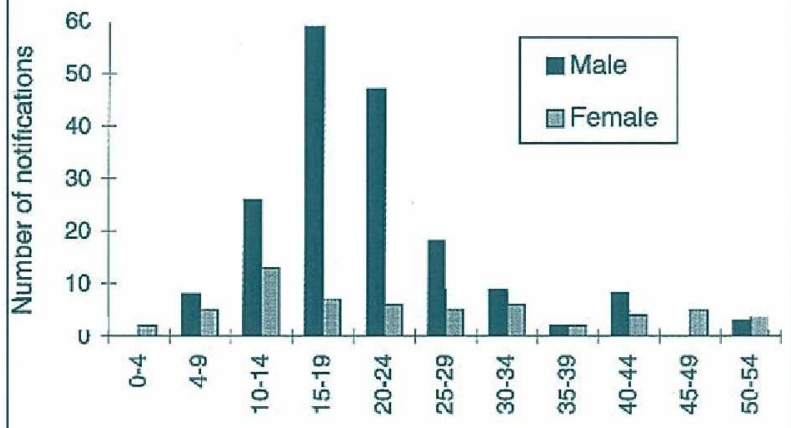

Age in years

expected following the introduction of measles-mumpsrubella (MMR) vaccination for all infants in 1989 (Figure 3). However, clinical reports during this outbreak suggested disease activity predominantly among young children of both sexes. This discrepancy casts some doubt on the representativeness of serologic notifications, and suggests that the NSW surveillance case definition may underestimate rubella occurring in young children. Clinicians are understandably reluctant to perform blood tests on young children with mild illness, and this may contribute to under-representation of children in rubella surveillance data. Conversely, adults, who tend to have more severe symptoms, may be over-represented because of more frequent serologic testing.

The preponderance of males among serologically diagnosed rubella cases reflects the older median age of this group. Young female adults are more likely to be immune because adolescent schoolgirls received rubella vaccination during the years 1971-1994. The sex distribution of clinical cases is more equal because most ( 70 per cent) are under 10 years old and had not been exposed to this differential immunisation.

This study has some important limitations. Occupation and language spoken at home were poorly recorded, so it is difficult to interpret these data confidently. The number of clinically diagnosed cases was small, and many of these may not have been rubella. Numerous viral illnesses can mimic rubella, and the specificity of a clinical diagnosis of rubella can be very poor in young children ${ }^{4,5}$. The positive predictive value of clinical diagnosis may have been somewhat improved because of greater rubella prevalence during this outbreak, but we were unable to evaluate this formally because clinical cases were not tested serologically. The fact that the majority of clinical cases occurred during AugustOctober, a few weeks before most serologic notifications, does support that many of these were caused by rubella.

To evaluate the appropriateness of rubella surveillance it is important to clarify its purpose. The primary objective of rubella control is to eliminate $\mathrm{CRS}^{6}$. This depends on two related immunisation strategies. The first is to ensure that women of childbearing age are immune. This is achieved by vaccinating adolescent females, and by post-natally vaccinating women who are non-immune during pre-natal screening. This strategy effectively reduces the incidence of CRS, but on its own does not appear to change the epidemiology of rubella among pre-adolescent women, children, and young men - who may serve as a persisting source of infection for pregnant women ${ }^{4,5,7}$. The second strategy is to protect pregnant women from exposure to rubella, by vaccinating all children and all adolescent $\mathrm{s}^{6}$. The primary outcome measure for these strategies, CRS, is notifiable in NSW and is also monitored by the Australian Paediatric Surveillance Unit. This enables us to monitor progress towards the elimination of CRS, but is insensitive to the short-term and medium-term effects of immunisation strategies on the dynamics of population immunity.

What, then, is the role of surveillance for incident rubella cases? It allows long-term trend analysis of age- and sexspecific incidence of rubella. Because all rubella vaccination is now performed using the combined MMR vaccine, future rubella surveillance will also give proxy information about those at risk for measles. As a surveillance definition serodiagnosis is highly specific, but it does not provide timely (or representative) data. In this study, the median time from onset of symptoms to notification was 19 days for serologic cases. Consequently, interventions for sporadic rubella are rarely possible in response to serologic surveillance, and outbreak recognition is likely to be delayed. The current requirement for school and child care centre-based reports causes some confusion instead of hastening intervention, because these cases also require serologic confirmation to satisfy notification criteria.

It is important to resolve whether acute interventions are an essential part of our long-term rubella control strategies. For this decision, some critical questions need to be answered. Does excluding rubella cases alone have any worthwhile impact on transmission? Should both children with rubella, and (as is recommended in the USA ${ }^{6}$ ) their unvaccinated contacts be excluded from school and child care? Is rubella outbreak control using targeted vaccination campaigns cost effective, or should resources be devoted to ensuring high rates of vaccination coverage at routine milestones? If it is decided that acute interventions are important, the NHMRC surveillance definition should be adopted. This incorporates a clinical component for contacts of serologically-confirmed rubella, and would enable more timely outbreak recognition, and appropriately targeted vaccination programs. Ideally, before adopting this definition in NSW we should formally evaluate its sensitivity and specificity, using serology as the reference standard. In practice it may be difficult to enrol young infants and children into a serodiagnostic study for very mild illness.

It is worth noting that other, more sensitive, rubella surveillance methodologies are possible using intermediate outcome measures. Intermittent population-based serosurveys, and continuous surveillance of antenatal screening allow detailed analyses of the relative influences of childhood, adolescent, and postnatal immunisation 


\section{Public HEALTH ABSTRACTS}

P ofessor James S. Lawson, Professor and Head of the School of Health Services Management at the University of NSW, has prepared the following public health items from the literature.

\section{EARLY INFANT DIETS AND INSULIN-DEPENDENT DIABETES}

A meta-analysis of epidemiological studies has shown a weak but significant association (odds ratio 1.5) between infant nutrition and the risk of insulin-dependent diabetes (IDD). This study showed that IDD patients were more likely to have had earlier exposure to cow's milk and were likely to have been breast fed for a shorter time than healthy individuals. Later investigations have not supported this finding. Accordingly, breast feeding should be supported for many good reasons and current infant feeding guidelines should not be changed until much more concrete evidence is available.

Ellis TM, Atkinson MA. Early infant diets and insulin-dependent diabetes. Lancet 1996; 347:1464-5.

\section{SALT-HIGH BLOOD PRESSURE LINK CONFIRMED}

An international study (Intersalt) has confirmed earlier work that gives further substance to the long-known link between consumption of high levels of salt and high blood pressure. In addition, a study in chimpanzees has shown that the addition of $100 \mathrm{mmol}$ of sodium to their diet increased their systolic blood pressure by $12 \mathrm{~mm} \mathrm{Hg}$. As three-quarters of the salt in the diet is hidden in processed food, a priority is to influence the food industry. As salt is the main source of "taste" this is an extremely difficult but worthwhile exercise.

Elliott $P$ et al. Intersalt revisited: further analyses of 24-hour sodium excretion and blood pressure within and across populations. Br Med $J$ $1996 ; 312: 1249-53$

\section{SIDS: RISE AND FALL ACCORDING TO SEASON}

The incidence of sudden infant death syndrome (SIDS) has fallen dramatically in recent years. This fall has been attributed to the encouragement of the infant sleeping face up and having infants sleep in the parents' bed or bedroom. However in both the UK and Australia, despite variations in climate temperature and the reduction in deaths, sudden infant deaths remain much more common in summer than in winter. This is unexplained but may be a clue to aetiology.

Douglas AS et al. Seasonality and the sudden infant death syndrome during 1987-9 and 1991-3 in Australia and Britain. Br Med J 1996; 312:1381-3.

\section{REGULAR EXERCISE IS OF EMOTIONAL BENEFIT TO TEENAGERS}

A prospective UK study has confirmed that there are benefits to the mental health of teenagers associated with regular physical exercise.

Steptoe A, Butler N. Sports participation and emotional wellbeing in adolescents, Lancet 1996; 347:1789-92.

\section{ALL TYPES OF ALCOHOLIC DRINKS REDUCE CORONARY HEART DISEASE}

The inverse association between alcoholic drinks and coronary heart disease is well established. A review of the studies strongly suggests it is the alcohol itself that is responsible for the reduction. Previously it has been thought it was other substances, including the ingredients of red wine.

Rimm et al. Review of moderate alcohol consumption and reduced risk of coronary heart disease: is the effect due to beer, wine or spirits? Br Med J 1996; 312:731-6.

\section{DRINKING THIAMINE TO HELP PREVENT ALCOHOL- RELATED BRAIN DAMAGE}

About 500 Australians develop brain damage each year because of alcohol consumption (Wernicke-Korsakoff syndrome). This brain damage is caused by the poor diets of many heavy drinkers which leads to thiamine deficiency. Since 1991 it has been compulsory for bread manufacturers to use thiamine-enriched flour as a public health measure against such brain damage. However enriching alcoholic drinks with thiamine is a much less expensive alternative by a factor of some 36 .

Connelly L, Price J. Preventing the Wernicke-Korsakoff syndrome in Australia: the cost effectiveness of thiamine-supplementation alternatives. Aust NZ J Public Health 1996; 20:181-7.

\section{Rubella outbreak in Western Sydney}

\section{Continued from page 71}

programs on population immunity ${ }^{4,8-10}$. In addition, surveillance of rubella occurring during pregnancy and of therapeutic abortions undertaken for intrauterine rubella, is more sensitive than counting cases of $\mathrm{CRS}^{4}$. None of these surveillance methods is employed in NSW. Their use should be considered.

\section{ACKNOWLEDGMENTS}

The authors thank A/Professor Margaret Burgess (New Children's Hospital, Westmead) for critically reviewing this report. The Master of Applied Epidemiology Program is funded by the Commonwealth Department of Health and Family Services.
1. New South Wales Health. Notification trends: Increase in rubella notifications. Public Health Bulletin 1995; 6:95-96.

2. NSW Health. Notification trends: Rubella. Public Health Bulletin $1995 ; 6: 152$

3. Center for Disease Control and Prevention. Epi Info 6.02. A word processing, database, and statistical program for Public Health. Atlanta, USA, 1994.

4. Miller E, Tookey P, Morgan Capner P et al. Rubella surveillance to June 1994: third joint report from the PHLS and the National Congenital Rubella Surveillance Programme. Commun Dis Rep CDR Rev 1994; 4:R146-52.

5. Herrmann KL. Rubella in the United States: toward a strategy for disease control and elimination. Epidemiol Infect 1991; 107:55-61.

6. Rubella prevention. Recommendations of the Immunization Practices Advisory Committee (ACIP) MMWR 1990- 39:1-18.

7. Miller E. Measles, mumps and rubella: present and future

immunisation policy. Public Health 1988; 102:317-321

8. Noah ND, Fowle SE. Immunity to rubella in women of childbearing age in the United Kingdom. BMJ 1988; 297:1301-1304.

9. Edmond $\mathrm{E}$, Zealley $\mathrm{H}$. The impact of a rubella prevention policy on the outcome of rubella in pregnancy. Br J Obstet Gyncecol 1986; 93:563-567. 10. Anderson RM, Grenfell BT. Quantitative investigations of different vaccination policies for the control of congenital rubella syndrome (CRS) in the United Kingdom. J Hyg Lond 1986; 96:305-333. 